\title{
A PLICACIÓN DE UNA METODOLOGÍA PROACTIVA DE CONTROL CONCURRENTE EN ENTIDADES PÚBLICAS
}

\section{APPLICATION OF A PROACTIVE METHODOLOGY OF CONCURRENT CONTROL IN PUBLIC ENTITIES}

\author{
${ }^{1}$ Luzmila Vera Flor
}

\section{RESUMEN}

Esta investigación se realizó con la finalidad de identificar la aplicación del Control Interno en las entidades del Estado, en cumplimiento con la disposición normativa según la Ley 27785, Ley orgánica del Sistema Nacional de Control y de la Contraloría General de la República (23/07/2002), para prevenir riesgos que afecten los objetivos institucionales y a fin de que se adopten las acciones correctivas que correspondan. Las Normas Generales de Control Gubernamental, aprobadas por R.C. No 273-2014-CG, disponen que el control simultáneo (Directiva No 017-2016-CG/DPROCAL, R.C. N ${ }^{\circ}$ 432-2016-CG), se puede llevar a cabo mediante otra modalidad de control denominado Control Concurrente para la Reconstrucción con Cambios - sustentado mediante la Directiva No $005-2017-C G / D P R O C A L$ y la R.C N ${ }^{\circ}$ 405-2017-CG - con el propósito de contribuir oportunamente con la correcta, eficiente y transparente utilización y gestión de los recursos y bienes del Estado. Asimismo, la Ley 30556, numeral 7.4, señala que los procesos de contratación de bienes, servicios, obras y consultorías ejecutados por las entidades públicas dentro del marco de la Ley 30225, Ley de contrataciones del Estado, están sujetos a procedimientos de control gubernamental, siendo uno de ellos el control concurrente; para tal efecto la Contraloría General de la República aprueba un Plan de acción de Control.

Palabras Clave: Control concurrente, entidades públicas, reconstrucción con cambios, riesgo.

\section{ABSTRACT}

This investigation was carried out to identify the application of Internal Control in State entities, in compliance with the normative provision according to Law 27785, Organic Law of the National Control System and the Office of the Comptroller General of the Republic (23/07/2002), to prevent risks that affect the institutional objectives, so that the corresponding corrective actions are adopted. The General Norms of Governmental Control, approved by R.C. No. 273-2014-CG, provide that the simultaneous control (Directive No. 017-2016-CG / DPROCAL, RC No. 432-2016-CG), can be carried out by another control method called Concurrent Control for Reconstruction with Changes, supported by Directive No. 005-2017CG / DPROCAL and RC No. 405-2017-CG, with the purpose of contributing in a timely manner to the correct, efficient and transparent use and management of State resources and assets. According to the law 30556, numeral 7.4, it authorizes, that the processes of contracting goods, services, works and consultancies executed by public entities within the framework of the law 30225, Law of contracting of the state, are immersed to procedures of governmental control, being one of them the concurrent control, for the effect the Comptroller General of the Republic approves a Control Action Plan.

Keywords: Concurrent control, public entities, reconstruction with changes, risk. 


\section{INTRODUCCIÓN}

La metodología proactiva del control concurrente no determina responsabilidades, sino más bien es un proceso positivo de identificación de eventos, o de riesgos, que asociados a una combinación de técnicas y procedimientos resulta accesible y sirve de motivación a los funcionarios y empleados públicos de las entidades para desarrollar sus funciones. De este modo, el cumplimiento del control interno se constituye en una actividad fundamental que busca garantizar una eficiente gestión y el uso correcto de los recursos del Estado dentro del marco legal.

En este contexto, surge una pregunta importante: ¿Cómo se dan cuenta los ciudadanos de que su dinero se gasta con eficiencia? El libro de Jorge Barajas Palomo (México) sirve para fundamentar la teoría y práctica de esta metodología.

El control concurrente, (aprobado mediante R.C N $\mathrm{N}^{\mathrm{O}}$ 405-2017-CG, directiva 0052017CG/DPROCAL) es una modalidad de control simultáneo que tiene su sustento normativo en la directiva $\mathrm{N}^{\mathrm{o}} 017-2016$ CG/DPROCAL RC N ${ }^{\circ}$ 432-2016-CG (27.02.18) con vigencia actual para las entidades públicas. Dicho control consiste en evaluar una o más tareas o actividades de un proceso seleccionado, con la intención de alertar oportunamente al Titular de la entidad y a las instancias competentes sobre la existencia de algunas situaciones adversas que afectan o podrían afectar el desarrollo de la entidad y/o el logro de los objetivos; es decir, esta evaluación se realiza a fin de que se adopten las acciones correctivas.

La reconstrucción con cambios (según Ley 30556) establece que las entidades se someterán al control gubernamental sobre las adquisiciones de bienes y servicios, contratación de obras, asesorías y consultorías; por ello se emitirá el Plan de Integridad de reconstrucción por la Autoridad, el Plan de Acción de Control y Directivas.

Actualmente todas las entidades públicas y privadas están expuestas a diferentes tipos de riesgos, por lo cual es necesario un buen sistema de control interno para minimizar riesgos; en consecuencia, se puede definir el riesgo como la posibilidad de que ocurra un evento adverso que afecte el logro de los objetivos de la entidad. (Coso ERM).

\section{OBJETIVOS GENERALES}

1. Proporcionar nuevos procedimientos y técnicas en el control concurrente para optimizar las actividades en las entidades del Estado.

2. Impulsar la capacitación teórica y práctica del nuevo contingente que interviene en la aplicación del control concurrente, dispuesto por el órgano de control.

3. Plantear una metodología práctica proactiva en la cual se aprecie que se lleva un control concurrente en el ciclo normal de operaciones.

\section{OBJETIVOS ESPECÍFICOS}

a) Informar sobre los riesgos significativos que afectan las obras públicas, aplicando procedimientos y técnicas eficaces.

b) Planificar la capacitación teórica práctica para los nuevos especialistas en el control concurrente, a fin de lograr la eficacia del control.

c) Diseñar el área de influencia del ciclo normal de operaciones que aborda las cuentas del activo corriente y pasivo corriente, en el periodo de un año, dentro del proceso de contrataciones $\mathrm{y}$ adquisiciones.

En esta fase de la planificación se demuestra que el control concurrente se ejecuta en un tiempo breve, además se identifica cuáles son los riesgos en las cuentas del ciclo normal de operaciones, se varían de acuerdo al tamaño de la entidad, el lugar de ubicación, el ámbito y área de influencia; en este caso, para dicho control, se debe presentar el análisis desde el área Financiera (según Riesgo ciclo normal de operaciones) y área No Financiera. (Según Riesgos en las Obras Públicas) como puede apreciarse a continuación:

\section{ÁREA FINANCIERA \\ A. CICLO NORMAL DE OPERACIONES}

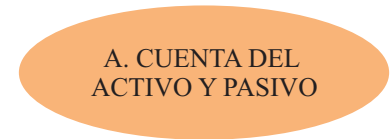

Figura 1. Ciclo normal de operaciones 
A. Identificación de riesgos en el ciclo normal de operaciones. Donde se define a las cuentas del activo y pasivo corriente sobre el movimiento de dinero y derechos dentro del periodo de un año, como son: caja bancos, inversiones temporales, cuentas por cobrar, almacenes y cuentas por pagar. Este ciclo sirve para demostrar si están documentados y autorizados, como se precisa en los siguientes elementos:

1. Pagos e ingresos a caja bancos sin el sustento documentario, ni la aprobación de las autoridades.

2. Cuentas por cobrar sin estructura financiera, direcciones domiciliarias no reales, y escasos análisis de cuentas.

3. Cancelaciones de adquisiciones de bienes de inversiones temporales, sin registro de cuentas por cobrar.

4. Omisión de toma de inventarios físicos de bienes y activos en el almacén.

5. Bienes cancelados para obras sin contabilizar el ingreso y salida en almacén.

6. Cuentas por pagar ocultas, registradas en cuentas de gastos para bajar resultados.

\section{ÁREA NO FINANCIERA}

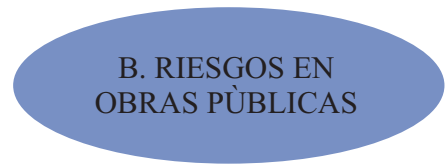

Figura 2. Obras públicas

A. Identificación de riesgos en obras públicas. Hace referencia a las obras que se ejecutan sin documentación saneada para no presentar liquidación final ni transferencia a los sectores que corresponde, como se puede apreciar en los siguientes elementos.

1. Obras sin expedientes técnicos, sin contratación de adquisición de bienes, ni servicios de consultoría.

2. Omisión de liquidación técnica y económica en la mayoría de las obras que acarrea mantenimiento en las entidades ejecutoras, motivo por el cual no se pueden transferir a los sectores que corresponde como son Salud, Educación, Transportes y otros.

3. En los contratos de obras, no se consideran las penalidades por atraso en las obras y/o abandono.

4. Obras no concluidas por falta de presupuesto.

5. Obras terminadas sin inventario físico ni liquidación técnica y económica.

6. Construcción de obras sin saneamiento físico y legal del terreno.

7. Paralización de obras según denuncias por irregularidades e intervención de la Contraloría.

\section{M P PROCEDIMIENTOS Y TÉCNICAS}

Dentro de la normatividad del control gubernamental, se habla sobre un proceso integral de control; es decir, para el control concurrente debe existir el proceso de planificación de las actividades a través de un plan y programa, luego la ejecución de dicho plan de actividades y posteriormente el informe final que alerta de posibles riesgos según las evidencias obtenidas. En consecuencia, para desarrollar esta acción se sugiere algunos procedimientos y técnicas como los que siguen a continuación:

1. Conocimiento del negocio, desde su origen es importante conocer las actividades de la empresa en todo su ámbito, el ambiente de control, el sistema de control, y lo más importante: la presencia de valores éticos que, sumados a la responsabilidad de los funcionarios y servidores, mediante un análisis deberán velar por el cumplimiento de objetivos de la entidad.

2. Aplicar la revisión, por medio de la implementación de los valores éticos en el ejercicio de los auditores y/o profesionales y especialistas para adicionar un valor agregado.

3. Inventarios de Riesgos, mediante un diagnóstico y análisis específico de riesgos de tipo legal, operativo y/o tecnológico se identifican riesgos en diferentes procesos de la entidad, siendo de preocupación su atención para minimizarlos.

4. Análisis Interno, a través de entrevistas, talleres de trabajo con personal de la entidad, 
clientes y proveedores, grupos de interés, expertos internos y externos, se va programando reuniones con fines de evaluación y de plantear las acciones correctivas.

5. Análisis de Flujo de Proceso, se considera la combinación de datos inputs, (entradas) tareas, y responsabilidades, además de los outputs (salidas) en las contrataciones de obras para identificar pagos mensuales, sin informe del supervisor de obra.

6. Talleres de Trabajo, utilizados de manera práctica para el trabajo en equipo y la optimización del tiempo con presencia del recurso humano, a fin de implementar los procedimientos y técnicas con el propósito de fortalecer la mejora en la gestión y cumplimiento de los objetivos institucionales.

7. Atención de Brechas, significa que en ocasiones existen brechas que retrasan el desarrollo de la entidad. Ejemplo de ello son los siguientes: a) Es evidente que los procesos operativos no están armonizados con los procedimientos y técnicas, notándose presencia de riesgos y debilidad en el control interno; b) El plan de acción de la Contraloría no está adecuadamente conciliado con el plan Integral de Reconstrucción con cambios, aprobado para atender casos de emergencia de la población afectada por El Niño Costero 2017; c) La capacitación integral para el personal de la entidad es escasa, se requiere demanda de tiempo y más presupuesto para la entidad. Todos estos hechos se tomarán en cuenta, en el momento oportuno, para que el responsable de la entidad tome las acciones correctivas que minimicen riesgos $\mathrm{y} / \mathrm{o}$ eviten posibles actos de corrupción.

\section{Evaluación de Técnicas}

Existen dos técnicas de evaluación:

A) Cuantitativas, cuando el riesgo no se puede cuantificar; es decir, no están disponibles los datos suficientes.

B) Cualitativas, aportan mayor precisión y se usan en actividades más complejas.

Estas técnicas constituyen una ayuda y una forma de visualizar el conocimiento; son la base para identificar riesgos que necesariamente tienen que ser evaluados por los especialistas para reforzar la aplicación del control interno. Aquí, algunas técnicas de uso:

1. La observación, para la evaluación de ciertas actividades y/o hechos presentados. Esta técnica es útil para el seguimiento frente a ciertas imprecisiones iniciales, con el fin de obtener la confirmación de alguna información y/o hecho que se busca aclarar, según precisan las normas generales de control gubernamental.

2. Procedimientos analíticos, son muy utilizados por diferentes especialistas de auditoría, para evaluar algunos saldos en las cuentas contables de la entidad y comprobar si los estados financieros son razonables.

\section{RESULTADOS}

Con el objetivo de concretizar esta investigación, se procedió a encuestar a profesionales de diferentes entidades públicas, categorizándolos en dos grupos:

Grupo A: Profesionales contadores y grupo B: Profesionales especialistas, como abogados, administradores, ingenieros, economistas. Esto con el propósito de obtener resultados mediante las siguientes interrogantes.

1. ¿Cree usted que es importante la implementación de los procedimientos y técnicas en el ejercicio del control concurrente para riesgos?

2. ¿Considera usted que la planificación integral se debe tomar en cuenta para usar oportunamente los recursos del Estado?

3. ¿Considera usted que es necesaria la capacitación teórica y práctica de los nuevos especialistas para la aplicación del control concurrente?

Respecto a la primera pregunta: ¿Cree usted que es importante la implementación de los procedimientos y técnicas en el ejercicio del control concurrente para riesgos?, ambos grupos opinaron que los procedimientos y técnicas a aplicarse varían para cada entidad, según su tamaño o área de influencia en el ciclo normal de operaciones. Pueden ser, por ejemplo, obras por contrata y/o administración directa - esto es según el segmento donde se aplique el servicio de Control Concurrente-, las cuales son importantes para acortar el tiempo una vez programadas, y actuar así de manera organizada; de otro modo no sería 
posible detectar los riesgos de valor y poner de conocimiento a las autoridades responsables para que tomen las medidas correctivas, sin perjuicio del control posterior.

Como se puede ver en la Tabla 1, ambos grupos coinciden; según el resultado, el $95 \%$ concuerda en que los procedimientos y técnicas son importantes para aplicar el control concurrente y además reducir el tiempo.

Tabla 1. Técnicas y procedimientos

\begin{tabular}{lcc}
\hline RESPUESTA & FRECUENCIA & PORCENTAJE \\
\hline SI & $\mathbf{1 9}$ & $\mathbf{9 5 \%}$ \\
NO & $\mathbf{1}$ & $\mathbf{5 \%}$ \\
TOTAL: & $\mathbf{2 0}$ & $\mathbf{1 0 0 \%}$ \\
\hline
\end{tabular}

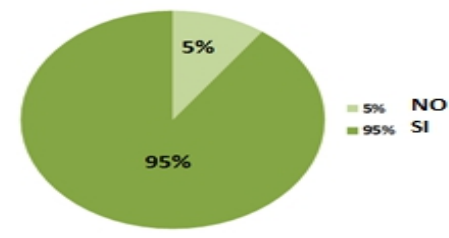

Figura 3. Técnicas y procedimientos

En cuanto a la segunda pregunta: ¿Considera usted que la planificación integral se debe tomar en cuenta para usar oportunamente los recursos del Estado?, el grupo " $A$ " opina que se pueden dar los casos de planificación Sectorial e Integral de acuerdo a las circunstancias, dimensiones y responsabilidades de las entidades ejecutoras (gobierno nacional, regional o local); esto asociado al plan estratégico aprobado para cada entidad, y a su vez, relacionado con los lineamientos considerados en el CEPLAN denominado Centro de Planificación Estratégica, creado con Ley 28522 de 6 de mayo de 2005, según Decreto Legislativo 1088. Es un organismo técnico especializado que ejerce rectoría de planificación en todas las entidades, encargado de formular, coordinar, dar cuenta y evaluar las estrategias principales de gobierno.

Por otro lado, el grupo "B" tiene una opinión más precisa y afirma que la planificación integral se define como la planificación y ejecución de obras, adquisición de bienes y servicios y consultorías en el menor tiempo; es más beneficiosa en costo beneficio y rapidez porque ahorra tiempo, y genera utilidad a la población que tanto lo necesita. A todo esto, se suma que hay coordinación mutua entre las autoridades regionales y distritales, plasmado en el trabajo en equipo, y todo ello en cumplimiento de las recomendaciones vertidas por la Contraloría.

Sirva para ilustración, el resultado mostrado en la Tabla 2, donde del total de 20 profesionales encuestados, el $90 \%$ casi coincide y afirma estar de acuerdo con la coordinación de los Planes de Control y resaltan que el Plan Integral es beneficioso para el avance de obras, de conformidad con la necesidad de población; mientras que el 10\% manifiesta que se debe revaluar los Planes para ser más realistas y así puedan solucionar las necesidades básicas de la población.

Tabla 2. Planificación integral

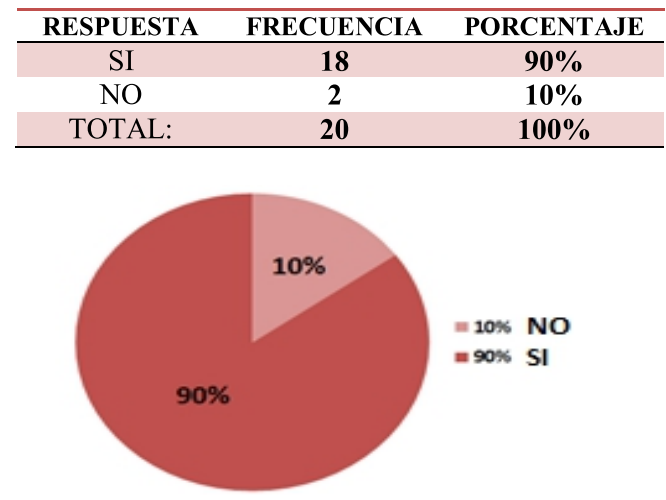

Figura 4. Planificación Integral

En referencia a la tercera pregunta: ¿Considera usted que es necesaria la capacitación teórica y práctica de los nuevos especialistas para la aplicación del control concurrente?, el Grupo "A" opina que la capacitación teórica práctica del personal técnico y funcionarios sobre el control concurrente es necesaria y oportuna; debe ser tomada en cuenta por la entidad para garantizar y obtener conocimientos e intercambio de experiencias prácticas con especialistas del órgano de control autorizados por la Contraloría, siendo ellos quienes evalúan la 
gestión al término de un ejercicio económico y presupuestal. Es necesario precisar que esta capacitación debe ser por grupos de especialidad, con el fin de que los empleados utilicen un solo criterio en la aplicación del control concurrente.

El Grupo "B" opina que la capacitación debe darse, en primer orden, sobre los instructores o profesores de auditoría, utilizando últimos procedimientos y/o técnicas con el propósito de llegar a una misma idea de control concurrente; por ello se plantea un programa de capacitación de tal modo que funcione la palanca intelectual, esto significa que la capacitación recibida en Lima también deberá ser igual en otras regiones del Perú. En consecuencia, la capacitación teórica y práctica es sumamente importante y urgente a nivel nacional, aplicable a todas las entidades del Estado debiendo plasmarse a través de un manual práctico.

En suma, ambos grupos -en el $75 \%$ de casos - opinan que la capacitación teórica y práctica es indispensable para tener éxito en el control concurrente; en tanto que el $25 \%$ de profesionales señala que es necesario tener una capacitación personalizada, asistiendo a una experiencia práctica de trabajo de campo. Véase estos resultados en la Tabla 3.

Tabla 3. Capacitación teórica y práctica

\begin{tabular}{lcc}
\hline RESPUESTA & FRECUENCIA & PORCENTAJE \\
\hline SI & 15 & $75 \%$ \\
NO & 5 & $25 \%$ \\
TOTAL & 20 & $100 \%$ \\
\hline
\end{tabular}

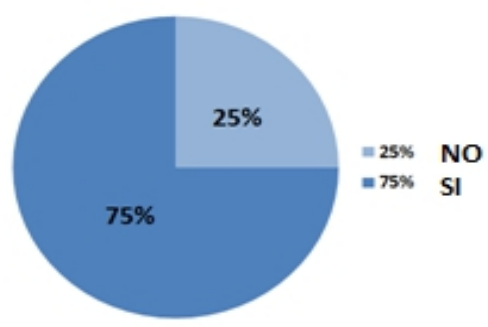

Figura 5. Capacitación teórica y práctica

\section{PROPUESTAS DE LA INVESTIGACIÓN}

Como resultado de esta investigación se propone tres aspectos fundamentales:

\section{A ) S O B R E E L C O N T R O L CONCURRENTE}

Para efectuar un servicio de control concurrente más efectivo, se propone la evaluación del Control Interno en su totalidad, tanto en el Área Financiera como en el Área No Financiera. Con el fin de garantizar que la información financiera sea confiable y libre de cualquier indicio de fraude o corrupción para la toma de decisiones, la primera área mencionada abarca el Ciclo Normal de Operaciones que viene a ser el activo corriente y pasivo corriente; mientras que en la segunda área se puede ver la realización del control concurrente a través de la ejecución de las obras públicas en distintas regiones a nivel nacional - según nota de prensa de la Contraloría, se indica que son 8302 obras distribuidas en 13 regiones afectadas por El Niño Costero en el año 2017-; las cuales deben ser evaluadas desde su fase de planificación, en cumplimiento a requisitos sobre licitaciones públicas y con el financiamiento presupuestal autorizado para el caso.

\section{B) SOBRE LA PLANIFICACIÓN INTEGRAL}

Para efectuar un servicio de control concurrente se propone la existencia de una Planificación Integral, porque de esa manera se avanzaría más rápido en la ejecución de obras, reduciendo el tiempo y costos; así se cumpliría mejor con el requerimiento de las necesidades básicas de la población.

La Planificación Integral es definida como una planificación de sectores para construir obras donde intervienen dos o más sectores, con casi el mismo expediente técnico; por tanto, resulta más beneficiosa. Por ejemplo: una carretera que une La Libertad, Lambayeque, Piura y Tumbes; lo mismo puede suceder con construcción de viviendas de diferentes sectores, entre otros casos.

\section{C) SOBRE LA CAPACITACIÓN TEÓRICAY PRÁCTICA}

Además de una planificación integral, como en el caso anterior, se propone tener una capacitación teórica y práctica permanente para el contingente nuevo que va a participar como especialistas contratados por la Contraloría, quienes aproximadamente varían entre $400 \mathrm{y}$ 4000 personas; es decir, según disposiciones o convenio a nivel nacional primero se debe entrenar a estos capacitadores para obtener una 
armonización única del control concurrente con un comportamiento técnico proactivo, a fin de detectar oportunamente eventos de riesgos y que sean comunicados a las autoridades responsables para que tomen las medidas correctivas.

Las propuestas mencionadas son viables, positivas, proactivas y flexibles conducentes para un mejor rendimiento que satisfaga a la entidad, y brinde beneficio a la población; lo cual constituye un aporte del presente estudio.

\section{BENEFICIOS DEL CONTROL CONCURRENTE}

Se detalla los beneficios de control concurrente como un valor agregado a las entidades, por comunicarse oportunamente e identificar los riesgos para su minimización, los cuales se detallan a continuación:

a) Es Proactivo, porque ayuda a la entidad y es oportuno para comunicar los riesgos.

b) Es Positivo, porque contribuye a administrar los riesgos.

c) Flexible, porque se adecúan a la aplicación en los procesos sin reparos.

d) No se determina responsabilidades de los funcionarios y servidores.

e) Es concreto, por comunicar los riesgos para que no se repitan y se logren los objetivos.

f) Muestra una situación de agilidad, es decir, no demanda mucho tiempo para detectar los riesgos.

g) Constancia, se deja por escrito la detección de los riesgos para superar las causas de los mismos.

h) Contribuye en la mejora de la gestión, pues ayuda a detectar indicios de corrupción en la ejecución de obras y/o adquisición de bienes y servicios.

i) Es meritorio, por contar con la presencia de auditores del sistema nacional de control como un medio de evaluación permanente en busca de la mejora de la entidad.

\section{REFERENCIAS BIBLIOGRÁFICAS}

Aguilar, J. (2008). Curso de Auditoría de gestión. Lima. Perú, 43.

Barajas, J. (1993). Auditoría con valor agregado, enfoque de Calidad total. México, 93.

Constitución Política del Perú. (1993). Art. 82. Contraloría General de la República, ente rector del Sistema Nacional de Control. Congreso de la República del P e r ú . R e c u p e r a d o d e : http://pdba.georgetown.edu/Parties/P eru/Leyes/constitucion.pdf.

Contraloría General de la República. Firma de Convenio Institucional para promover la participación ciudadana en la Reconstrucción con Cambios. Nota de prensa No 122. (2017, 14 de noviembre) Lima. Perú. Recuperado d

http:www.contraloría.gob.pe/wps/wc $\mathrm{m} /$ connet/cgrnew/as_contraloria/pren sa/notas_de:prensa/2017/lima/np_12 2-2017-cg.

Gestión de Riesgos Corporativos (2009). Marco Gestión integral de Riesgo ERM (COSO 2). Lima, Perú. $\mathrm{R}$ e c u p e r a d o d e : http://www.econ.unicen.edu.ar/attach ments/2009_MaterialIERM.pdf.

Ley Orgánica del Sistema Nacional de Control y de la Contraloría General de la República No 27785. Art. 14,16, 22. (2002, 23 de julio) Lima, Perú.

Normas Legales Diario Oficial El Peruano, 226885. Recuperado de: http://www.leyes.congreso.gob.pe/Do cumentos/Leyes/27785.pdf.

Ley que dispone la creación de la autoridad para la reconstrucción con cambios. $\mathrm{N}^{\circ}$ 30556. Art. 7 (2017, 29 de abril) Lima, Perú. Normas Legales Diario Oficial El Peruano, 4. Recuperado de:busuedas.elperuano.pe/download/f ull/9xgDinpfaAB96ZaN572UQT. 
Ley del Congreso de la República $N^{\circ} 28522$ crea el CEPLAN el 6 de mayo 2005. Lima Perú. Recuperado de: https://www.ceplan.gob.pe/wpcontent/uploads/2017/08/LEY-N28522.pdf.

Resolución de Contraloría N $N^{\circ} 361$ (2017). Aprueba lineamientos de política para el planeamiento del control gubernamental. Lima. Perú, 1. R e c u p e r a d o d e : http://doc.contraloria.gob.pe/transpar encia/documentos/2015/RC_361_20 15_CG.pdf.

Resolución de Contraloría $\mathrm{N}^{\circ}$ 273. Normas generales de control gubernamental. (2014,13 de mayo). Lima. Perú. Normas legales. Diario oficial El
Peruano. Recuperado de: http://www.munizlaw.com/normas/20 $14 / \mathrm{M}$ a $\mathrm{y}$ o / $13-05$ 14/SEPARATA\%20ESPECIAL.pdf.

Resolución de la Contraloría $N^{\circ}$ 405(2017) aprueba la Directiva $\mathrm{N}^{\circ} 005-2017$ CG/DPROCAL de 4 de noviembre de 2017, Control Concurrente para la Reconstrucción con cambios (2017, 04 de noviembre de 2017). Lima, Perú, 7. R e c u p e r a d o d e : https://www.mesadeconcertacion.org. $\mathrm{pe} / \mathrm{sites} / \mathrm{default} /$ files/archivos/2017/d ocumentos/11/resolucion_de contral o r i a n o . $4055-2017$ cg_aprobacion_de_directiva_0052017-cf.pdf. 